\title{
Chapter 9 \\ Dynamic Behavior of Bearings on Offshore Wind Turbine Gearboxes
}

\author{
Rubén Cerdá, Bart Blockmans, Jakob Fiszer, Tommaso Tamarozzi, \\ Bert Pluymers, and Wim Desmet
}

\begin{abstract}
Gearbox failure is among the highest causes of downtime in a wind turbine, causing a significant loss to the wind energy sector, especially in the complex offshore environment. Quite often, the cause of these gearbox and drivetrain errors, as well as other undesired noise and vibrations issues, is premature bearing failure. Therefore, developing more efficient and reliable bearing models and simulation methods that can accurately predict the nonlinear dynamic loads already in the design phase is still crucial. Without claims of completeness, a few important items to be considered when analyzing bearings and a state-of-the art review for bearing modelling approaches (from analytical lumped parameter models to complex flexible multibody simulations) will be discussed in this chapter. Furthermore, some recent modelling developments and the problem of integrating these bearing models with similar advanced gear models into flexible multibody simulations at full-scale wind turbine drivetrain level will be addressed.
\end{abstract}

\subsection{Introduction}

Improving the reliability of wind turbines (WT) is an essential component in the bid to minimize the cost of energy (COE) from wind, especially for offshore wind turbines because of the difficulties associated with access for maintenance. Unfortunately, many drivetrains are suffering premature bearing failures. These bearing failures are very costly, particularly given the long downtime incurred per failure. Numerous studies have been undertaken to obtain the distribution of failures by assembly in WTs. Gearbox and generator failure rates remain unacceptably high. Furthermore, the downtime for these failures is amongst the highest of all WT assemblies. Often the entire gearbox or generator needs to be replaced, which requires the deployment of a large crane. These cranes are costly and may take days or weeks to deploy. Offshore, it is not unusual for rough weather to prevent access

R. Cerdá $(\bowtie) \bullet$ B. Blockmans • J. Fiszer • T. Tamarozzi $\bullet$ B. Pluymers $\bullet$ W. Desmet Department of Mechanical Engineering, KU Leuven, Celestijnenlaan 300c, 3001 Leuven, Belgium e-mail: ruben.cerda@kuleuven.be; bart.blockmans@kuleuven.be; jakob.fiszer@kuleuven.be; tommaso.tamarozzi@kuleuven.be; bert.pluymers@kuleuven.be; wim.desmet@kuleuven.be 


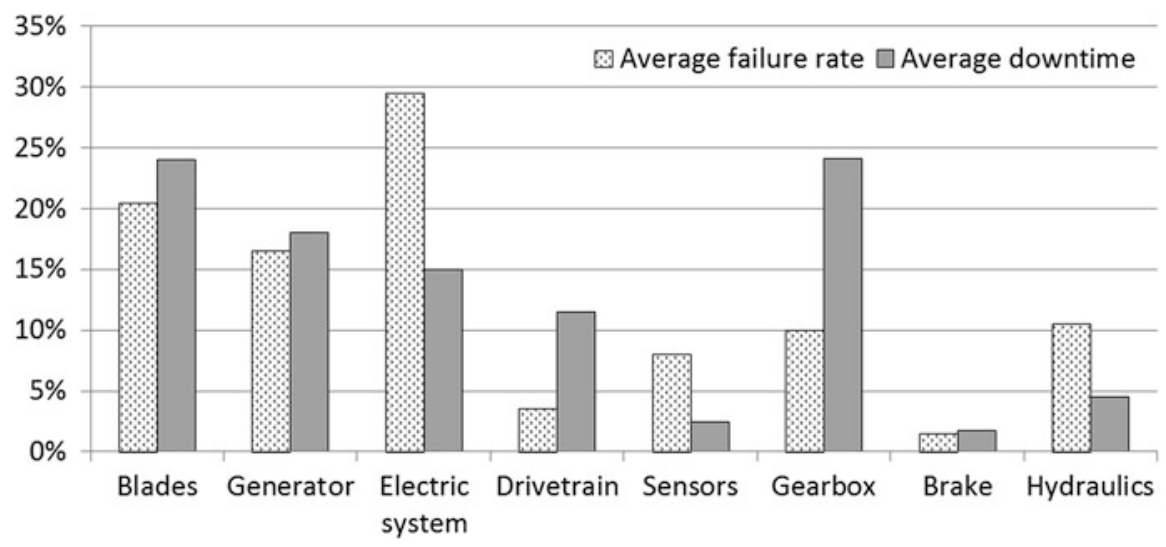

Fig. 9.1 Distribution of failures and downtime by assembly in WT (adapted from EWEA 2011)

for operation and maintenance for weeks at a time. Figure 9.1 shows the average failure rate and the incurred average downtime by assembly, based on the work presented in EWEA (2011).

Moreover, Chen and Alewine (2010) published the findings of a survey of over 800 failed WT generators, which showed that the dominant source in multimegawatt WT generators is the bearing. Despite this evidence, and although the unique operational conditions of the bearings resulting from widely varying wind loads and high vibration levels are well known, the root causes of these failure data are not completely known. There is, therefore, a need to search for more accurate and trustworthy component-level models and to undertake full dynamic multibody simulations at WT drivetrain system level.

Mechanical transmissions have been continuously attracting the interest of researchers and engineers, who dedicated consistent resources and efforts since the second half of the past century to investigate the multiple and interconnected phenomena that are typical in transmission dynamics. However, despite extensive studies in the last decades, system-level dynamics for the whole mechanical transmission is gaining a game-changer role and requires a deeper understanding and a more detailed knowledge to allow designers to address at the same time efficiency, durability and noise predictions and optimization.

Current simulation techniques rely on the assumption that the components are rigid, or adopt linearized modal reductions, which are applicable for the shafts and the housing but introduce significant approximations for the other components since they do not allow modelling the nonlinear dynamic contact interactions involved in the gear meshing and in the bearings with sufficient accuracy if a lumped-parameters approach is used.

Due to the highlighted reasons, the design of a WT drivetrain that is optimized from the dynamic point of view at a system level can be achieved only by establishing a simulation methodology that is capable of capturing, in a multi-discipline 
integrated, detailed yet computationally efficient way, all those phenomena that are relevant for the non-linear dynamic behavior of the system.

\subsection{Bearings for Wind Turbine Applications}

Besides gears, bearings are the other main sub-system in every mechanical transmission. Due to the great variety of bearing types, the scope of this section is narrowed down to rolling-element bearings (also known as rolling or roller bearings). This type of bearing is one of the most used in mechanical applications, playing a crucial and effective role across various industries such as automotive or wind turbines.

In this section, some of the main phenomena affecting bearings, with a specific focus on wind turbine applications, are discussed.

\subsubsection{Rolling-Element Bearings: Basic Concepts}

The term "rolling" is collectively used for all forms of bearings that utilize the rolling action of balls or rollers to minimize the friction from a constrained motion of one body relative to another. Most bearings provide a close relative position of two loaded components yet allow a rotational motion between them. Special types only allow a linear motion in the direction of a stationary shaft (e.g. linear guides). A combination of both degrees of freedom is also possible. Various types of rolling elements are available to achieve this constrained motion. They can be categorized into five standard shapes: ball bearing, cylindrical bearing, tapered bearing, needle bearing and barrel bearing; each suitable for a different application.

A common rolling element bearing typically consists of an inner and an outer ring and number of rolling elements which form the connection between the rings. The rolling elements are often guided by a cage to ensure uniform distribution and to prevent mutual contact. To prevent axial motion of the elements, both the inner and outer ring of most ball bearings include a gap called groove or raceway. Besides constraining the axial motion, the raceways also store the lubrication, which is necessary in order to reduce the friction and wear in the rolling contacts. A seal is also implemented to contain the lubricant and protect the raceway from dirt.

A more extensive version of this brief introduction to the nomenclature of rolling bearings can be found in Harris and Kotzalas (2006) and Wensing (1998).

The need to reduce vibrations of rotating machinery has stimulated researchers for many years to analyze the dynamic behavior of rolling element bearings. As the bearings are in the transmission path of vibrations between the shaft and bearing housing, they play a key role in the resulting vibrations. They determine for instance the machine's critical speeds and the forces acting on the different components. Subsequently, they influence the noise emanating from the machine and component 
stresses and wear. Some of the main phenomena that affect the non-linear dynamic behavior of the bearings are discussed in the following subsections.

\subsubsection{Contact Mechanics}

When two elastic solids contact under a load $w$, a contact area develops. In many engineering applications such as rolling element bearings and gears, the contact area is small and the resulting pressure is high. The local deformation and stresses can be determined from analytical formulas, based on the theory of elasticity and derived by Hertz. Hertz's contact model is based on the following assumptions (Wensing 1998):

- The material deformation is elastic.

- The load is directed normally to the contacting surfaces, such that surface shear stresses can be neglected.

- The dimensions of the contact area are small compared to the radii of curvature of the contacting bodies.

- The effect of surface roughness is negligible.

For a point contact in ball bearings, an elliptical shape of the contact area is assumed. Figure 9.2 shows the contact geometry (Jacobs 2014). The rolling element load $w$ is oriented in the $\mathrm{x}$-direction, the rolling direction equals the $\mathrm{y}$-direction and the bearing's rotation axis is oriented in the z-direction. The contact is characterized by its radii of curvature in two principal planes, perpendicular to each other. The first principal plane (xz-plane) contains the bearing's rotation axis and a rolling element center. The semi-major axis of the elliptical contact $a$ is located in this plane. The

a

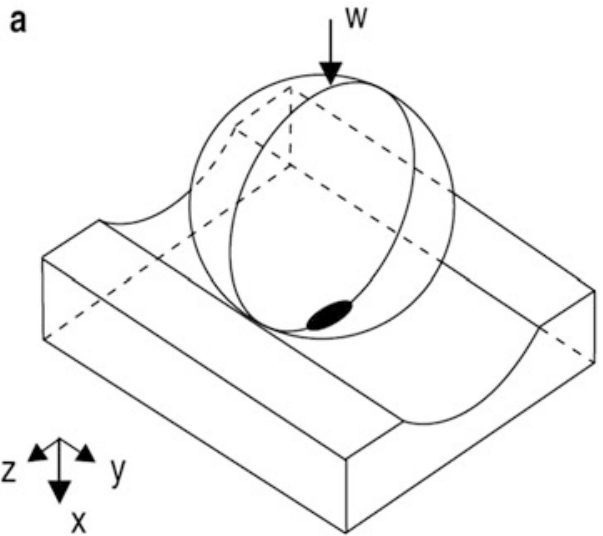

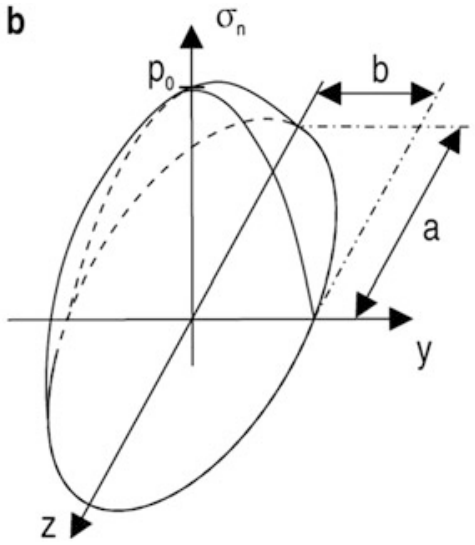

Fig. 9.2 (a) Elliptical contact area in black and in (b) the corresponding surface stress distribution (Jacobs 2014) 
second principal plane (xy-plane) is perpendicular to the rotation axis and contains a rolling element center. The semi-minor axis of the elliptical contact $b$ is located in this plane.

The contact problem is reduced to the problem of a paraboloid shaped surface approaching a flat surface. The normal stress is distributed over the contact area in the form of a semi ellipsoid, as shown in Fig. 9.2b. The maximum normal stress at the surface $p_{0}$ occurs at the geometrical center of the contact area and thus amounts to 1.5 times the evenly distributed pressure:

$$
p_{0}=1.5 \frac{w}{A}
$$

where the contact surface area $A$ is given by:

$$
A=\pi \cdot a \cdot b
$$

with $a$ and $b$ being the semi-major and semi-minor axis of the elliptical contact.

This maximum stress is also called maximum Hertzian contact pressure. The normal stress $\sigma_{n}$ at all other points within the contact area is given by:

$$
\sigma_{n}=p_{0}\left(1-\left(\frac{z}{a}\right)^{2}-\left(\frac{y}{b}\right)^{2}\right)^{1 / 2}
$$

Beside the surface stresses caused by a concentrated force applied perpendicular to the surface, rolling contacts of ball bearings also have subsurface stresses. The principal stresses occurring below the center of the contact area were calculated by Jones (1946). Based on Mohr's circle, the maximum shear stress is derived as well. Due to friction between the rolling elements and raceways, tangential forces are introduced on the surface. The effect of the resulting surface shear stress on the subsurface stresses was analyzed by Zwirlein and Schlicht (1980).

\subsubsection{Influence of the Lubricant Film}

The first realistic model which provided an approximate solution for the lubricant film thickness was proposed by Grubin (1949). It was found that the combination of three effects is instrumental to the lubrication mechanism: hydrodynamic lubrication, elastic deformation of the metal surfaces and the increase in viscosity of oil under extreme pressures. It was shown theoretically that under the condition of intense contact stress, a lubricating oil film can be formed. This elastohydrodynamic lubrication (EHL) film is very thin $(0.1-1 \mu \mathrm{m})$, but separates the interacting surfaces.

In a stationary contact between a ball and raceway, the pressure distribution is ellipsoidal according to the classical Hertzian theory (Fig. 9.2b). The pressure field 
Fig. 9.3 Pressure distribution considering the EHL film (Jacobs 2014)

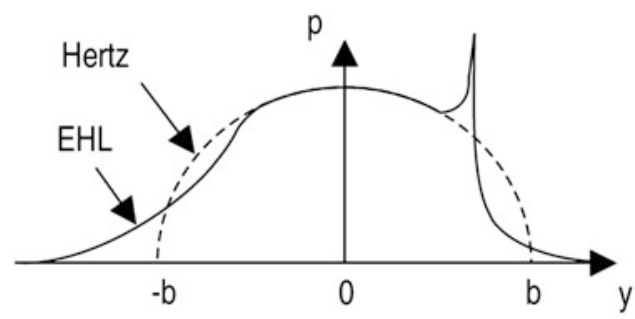

changes when the lubricant is dragged into the contact with a mean velocity. An EHL film is generated, which modifies the pressure distribution at the entry and exit regions of the contact. Figure 9.3 (Jacobs 2014) shows the pressure distribution along the minor axis of the elliptical contact.

Due to the strong interaction between the EHL film and the deformation of the contacting surfaces, the lubricant film affects the stiffness and damping characteristics of the bearing.

One of the most up-to-date analyses of the stiffness and damping evaluation for elasto-hydrodynamic contacts is based on the assessment of the dynamic response through a full dynamic simulation of the contact forces. It was first published by Wijnant (1998) and later extended by Wensing (1998) and Nonato and Cavalca (2012). Owing to the formation of a lubricant film, an increasing stiffness of each contact in comparison to a dry contact is observed. Also, the effective internal bearing clearance is reduced. The formation of a lubricant film consequently leads to an increased bearing stiffness.

Experimental studies analyze the variations of the bearing dynamics for different values of the load, speed and lubricant's viscosity. Dietl et al. (2000) measure the damping capabilities of two rolling element bearings supporting a rigid shaft. Mitsuya et al. (1998) investigate the damping characteristics of a single deep groove ball bearing. A higher damping of the lubricated bearing in comparison to a dry bearing is observed in each publication. The difficulty of performing sufficiently accurate measurements and the multitude of parameters influencing the results (type of bearings, radial and axial preload, speed, loose/interference fits of the bearing seats, lubricant properties, temperature, etc.) is emphasized in most of these publications.

\subsubsection{Durability}

Rolling elements bearing failure is one of the foremost causes of breakdown in rotating machinery ( $\mathrm{Li}$ et al. 1999). Bearings fail prematurely in service due to phenomena like contamination, poor lubrication, poor fits, misalignments, etc. The main contributors to the decrease in the durability of a rolling bearing are the behavior of the lubricant film during strong external excitation and the damage of 
the raceway surfaces due to the external dynamic loads that can be applied to the bearing for a long period of time.

Common Fatigue Failure Modes Every rolling element bearing rotating under load above the endurance strength limit has a definite fatigue life. The bearing approaches the end of its life when the rolling surfaces are damaged by rolling contact fatigue (RCF). Two mechanisms of fatigue damage initiation can be distinguished (Muro and Tsushima 1970):

- The subsurface failure mode: In many applications, the lubrication of the rolling contacts is adequate and the friction between the rolling elements and raceways is negligible. The von Mises equivalent stress reaches its maximum at a point below the surface. The stress can locally exceed the yield strength of the bearing steel (between 1400 and $1800 \mathrm{MPa}$ ) such that plastic deformation occurs. During cyclic loading, residual stresses build up and minute cracks are formed below the surface. This is often observed in the region of material inhomogeneities, where the stresses are maximal. The subsurface cracks eventually progress towards the surface. Surface material can break loose such that deep spalls are formed. During the remaining bearing lifetime, the damage spreads rapidly since tornoff particles are rolled over causing local overloading. A detrimentally preloaded bearing, for instance due to poor fits or misalignment, fails prematurely due to RCF initiated below the surface.

- The surface failure mode: Ceramic or metallic foreign solid particles contaminate the lubricant in the contacts. The solid particles originate for instance from the grinding process of the bearing and/or its surrounding structure (carbide cluster). Due to over-rolling during the bearing operation, indentations on the raceway surfaces are formed. The Hertzian micro-contacts between the surfaces and the solid particles are subjected to cyclic loading. This results in increasing residual stresses near the surface up to a depth related to the size distribution of the indentations. Strain hardening by severe plastic deformation leads to material embrittlement and subsequent crack initiation on the surface. Further failure development produces shallow V-shaped pits which are originally only several micrometers deep. The pits are formed behind the indentations. Chips of surface material flake off causing accelerated wear. While a small amount of wear is natural and unavoidable in most bearing arrangements, inadequate sealing and defective lubrication lead to severe wear. The bearing fails prematurely due to RCF initiated at the surface.

Lifetime Calculation Accurate prediction of the lifetime of rolling element bearings is a crucial step towards a reliable design of many rotating machines. The general lifetime calculations are based on the assumption that the rolling surfaces flake sooner or later due to RCF initiated below the surface (subsurface failure mode). According to the classical fatigue theory, the first cracks usually emanate from inhomogeneities in the material, such as microscopically small inclusions or metallurgical dislocations. In recent years, inhomogeneities in the bearing material became less important as a cause of fatigue damage, owing to the 
constant improvement of the material cleanliness. Today, most cracks emanate from indentations caused by solid particles (surface failure mode). The inhomogeneities are randomly distributed in the material and vary in size and type. The solid particles may enter rolling contact areas at an earlier or later time. With identical bearings and operating conditions, fatigue damage therefore occurs after different periods of operation. Brandlein et al. (1999) describe a series of lifetime tests. Thirty bearings of the same batch are loaded in the same way till failure. Differences in lifetime up to a factor 20 are observed.

The wide scatter of running times explains the impossibility to accurately predict the lifetime of an individual bearing. Predictions can only be made for a large group of nominally identical bearings stressed in the same manner. The rating life $L_{10}$ is defined as the running time at which $10 \%$ of the bearings have failed. The ISO 281 standard gives the general equation for lifetime calculations, used for bearing dimensioning (ISO 2007):

$$
L_{10}=\left(\frac{C}{P}\right)^{p}
$$

where $P$ is the equivalent dynamic load which takes into account both the radial and axial bearing load. The dynamic load rating $C$ corresponds to a pure radial constant load at which the $L_{10}$ life equals one million revolutions. The exponent $p$ equals 3 for ball bearings. The rating life $L_{10}$ is expressed in million revolutions.

When a bearing is not stressed beyond its endurance strength limit and the operating conditions meet certain prerequisites with regard to the lubricant film thickness and cleanliness of the lubricating gap, it does not fail. Also, the bearing life not only depends on the load. Other influences, such as the lubrication condition, have a significant effect. These aspects are not taken into account by Eq. (9.4). However, the equation remains the basis of bearing dimensioning and the starting point for adjusted calculation methods. Since the 1970s, empirical factors are added to the equation, considering the endurance strength limit, the lubrication condition, etc. In many bearing arrangements, the load and rotational speed change either randomly or according to a work cycle. An equivalent dynamic load is then calculated based on a series of individual loads and speeds of a certain duration $q_{i}$. This calculation is derived from the Palmgren-Miner rule of variable amplitude loading in the classical fatigue theory. With the rotational speed $n_{i}$ when the load $P_{i}$ is acting on the bearing and the mean rotational speed $n_{m}$, the equivalent dynamic load equals:

$$
P=\left(\frac{\sum_{i=1}^{k} n_{i} q_{i} P_{i}^{p}}{n_{m}}\right)^{1 / p}
$$


Effect of External Dynamic Loads For bearings subjected to highly varying loads, recent research emphasizes a strong reduction of the actual bearing lifetime w.r.t. the calculated bearing lifetime. The influence of external dynamic loads on the lifetime of bearings is elaborately analyzed by Gegner (2011), and Nierlich and Gegner (2011). Cylindrical roller bearings are tested under high dynamic load using a novel bearing test rig. Due to axial vibrations, asperities of the contacting bodies experience strong sliding motion. Material aging is shifted towards the surface. This type of damage is not covered by the classical bearing lifetime calculations. Reductions of the actual $L_{10}$ bearing life of $80 \%$ w.r.t. the calculated lifetime are estimated.

\subsubsection{Non Torque Loading}

Non-torque loads include pitching moments caused by the rotor weight and tower shadow, wind-induced moments, moments caused by the controller, thrust, etc. Guo et al. (2014) and Park et al. (2013) have investigated the effects of non-torque loads, gravity and bearing clearance in the load-sharing and planetary loads of a threepoint suspension wind turbine drivetrain. Non-torque loads affect gear and bearing loads and planetary gear load sharing. It is present on all the different drivetrain configurations: 3-point suspension (Fig. 9.4), 2-point suspension and on integrated gearboxes.

An analytical formulation was developed and a 3D dynamic drivetrain model that included mesh stiffness variation, tooth modifications and gearbox housing flexibility was built (Guo et al. 2014).

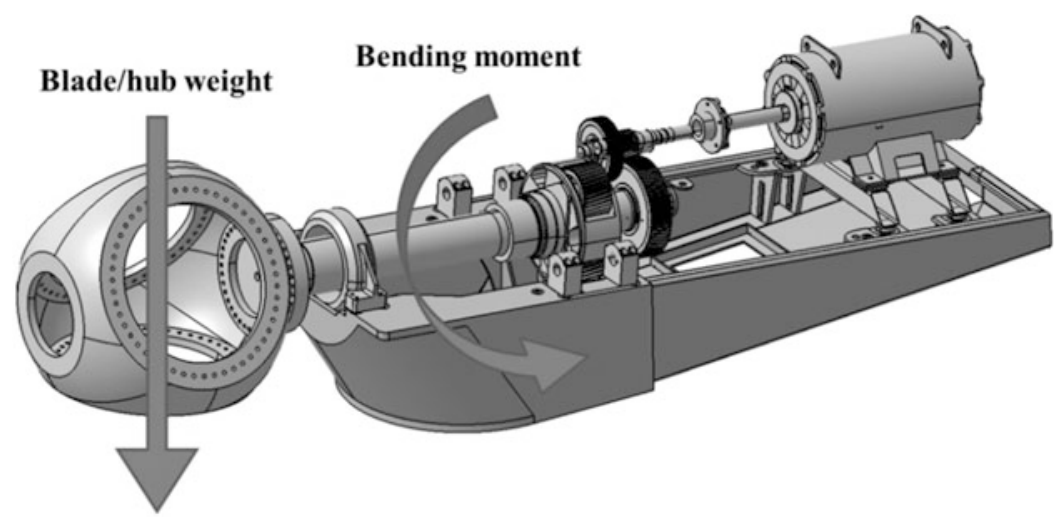

Fig. 9.4 Non-torque loadings and their effects on a typical 3-point suspension drivetrain 
The shaft pitching moment causes unequal load sharing between the upwind and downwind planet bearings, which can lead to abnormal tooth contact (consisting of tooth-edge loading, partial contact loss and reversing contact). These conditions can result in tooth failure modes such as micro-pitting. At low-input torque, the effects of non-torque loads on the gearbox internal loads were dominant. In this condition, the planet bearings are at risk of skidding and the gear teeth are at risk of contact loss, also leading to reduced gearbox life. The planetary load-sharing factor increased with bearing clearance. With carrier bearing-clearance, the shaft pitching moment was transmitted into the gear meshes and planet bearings. Optimizing the carrier-bearing clearance reduced the gearbox sensitivity to non-torque loads.

\subsection{Numerical Modelling of Bearings}

Modelling and simulation methods provide an increasingly accurate approach for predicting the dynamic performance of mechanical systems. The bearing models evolved from analytical formulations of a nonlinear spring to complex flexible multibody simulations. An overview of these modelling approaches is given in Sect. 9.3.1, while the technical details of the various approaches are discussed in Sects. 9.3.2-9.3.4.

\subsubsection{Overview of State-of-the-Art Bearing Models}

A bearing is a mechanical component that constraints the relative motion between two bodies by allowing relative rotation about one axis and constraining relative motion along the other coordinates (i.e. two relative rotations and three relative translational displacements). Due to the finite stiffness of a mechanical bearing and the presence of clearance, however, relative motion along the constrained coordinates can occur in practice when the bearing deforms or moves through its clearance. As such, a mechanical bearing can be viewed as a multi-dimensional spring with non-linear (state-dependent) stiffness constants. In this representation, the non-linearity of the spring is caused by clearance, the varying number of rolling elements in contact and the nonlinear nature of these contact interactions (see Sect. 9.2.2).

The representation of a mechanical bearing as a multi-dimensional spring has proven to be a productive ground for modelling bearings in modal analyses of multibody systems (Peeters 2006; Helsen 2012). As such analyses are typically conducted at a (or a sequence of) predefined operating point(s) of the multibody system, the non-linear bearing can be linearized and represented by a threedimensional linear spring. This spring is mathematically described by a constant symmetric 6-by-6 stiffness matrix (typically provided by the bearing manufacturer), which represents the relationship between the relative motion of the bearing's inner 
and outer rings and the resulting bearing forces. However, as this representation is only valid at a specific operating point, it cannot be used for time domain simulations when the nonlinear bearing stiffness and/or internal bearing dynamics are expected to play an important role in explaining the overall system behavior. In the latter case, non-linear bearing models should be used.

A first distinction in non-linear bearing modelling is based on the dimensionality of the problem: if only in-plane motion is considered, two-dimensional bearing models are to be used. These models allow relative rotation about the axis perpendicular to the plane, while constraining translational motion in the plane (note that for modal analyses, a linearized 2D bearing model represented by a 3by-3 stiffness matrix usually suffices). Three-dimensional bearing models are used when general motion is considered; in this case two relative rotations and three relative translations are constrained by the bearing model. Two-dimensional bearing models are discussed in Sect. 9.3.2, whereas three-dimensional models are discussed in Sect. 9.3.3.

A second distinction is based on the level of complexity and detail included in the flexibility description of the bearing. In Sects. 9.3.2-9.3.3, it is assumed that the (elastic) deformation of the bearing is confined to the contact zones within the bearing (i.e. the areas over which contact takes place between the rolling elements and the inner and outer raceways). Under this assumption, Hertzian contact theory (cf. Sect. 9.2.2) suffices to describe the elastic deformation of the bearing and to compute bearing stiffness. While the assumption of localized deformation is reasonable in a wide range of applications as far as the (generally compact and solid) rolling elements are concerned, this might not be the case for the bearing's inner and/or outer rings, especially when they are integrated with the bearing's flexible supporting structure (e.g. gearbox housing). In that case, Hertzian contact theory only describes the local contribution to the overall bearing deformation, whereas more advanced methods are required to describe the global (or 'bulk') bearing deformation. Such methods are described in Sect. 9.3.4.

Further distinctions of the various bearing models are based on the following aspects:

- Type of bearing: typically, a distinction is made between ball bearings (point contact between rolling elements and raceways) and roller bearings (line contact between rolling elements and raceways), because of the different mathematical tools required to model the contact interactions.

- Inclusion of dynamic effects such as centrifugal, gyroscopic and inertial effects: these effects can either be taken into account quasi-statically (excluding inertial effects) or by assigning a dynamic degree of freedom to each rolling element in the bearing model. In quasi-static bearing models the solution to a set of nonlinear algebraic equilibrium equations is generally required, while in dynamic models the solution is obtained through integration of the differential equations of motion of the bearing elements. Obviously, the importance of dynamic effects increases as the bearing's cage velocity increases. 
- Inclusion of the influence of elasto-hydrodynamic lubrication (EHL): lubrication reduces friction and wear in the contact interactions between rolling elements and raceways. The stiffness and damping properties of the EHL film play an important role in the transfer of mechanical vibrations through the bearing, and should therefore be taken into account when investigating internal bearing dynamics. As damping in the EHL film is mainly attributed to viscous losses in the lubricant (Wensing 1998), the damping behavior of the lubricant is typically taken into account by adding a linear viscous damper in parallel with the nonlinear contact stiffness spring. Wijnant (1998) obtained an analytical expression for the EHL damping constant in ball bearings by solving (for various parameters) the structural and fluid equations governing the transient behavior of a single EHL contact in which an initially equilibrated rolling element is slightly lifted from its equilibrium position and subsequently released. Similar analyses have been conducted for roller bearings (Qian 2013). Furthermore, the stiffness of a fully-developed lubricant film generally is several orders of magnitude larger than the Hertzian contact stiffness and therefore its contribution to the flexibility of the contact zone can be neglected.

- Inclusion of the cage: the rolling elements are usually held in an angularly spaced relationship by a cage or separator, which is generally constructed using materials that are relatively soft as compared to the rolling element and ring materials. A properly designed cage is often crucial for the bearing to withstand misalignment loads, and can therefore play an important role in establishing equilibrium of the bearing components. Cage modelling varies from (implicitly defined) geometric constraint relations to the inclusion of flexible cage representations including contact modelling of the interactions between cage and rolling elements.

- Inclusion of the influence of friction: friction in a bearing is made up of several components, including rolling friction, sliding friction of both the rolling elements and the cage, fluid friction and seal friction. These components lead to (motion-resisting) frictional forces and moments in the bearing that can modify the equilibrium conditions in the bearing. Friction energy is converted to heat, which is mostly dissipated by the (preferably recirculating) lubricant. Friction in particular plays a role in cylindrical and tapered roller bearings, where sliding contact between the roller ends and ring flanges can result in considerable frictional forces.

In the subsequent sections, the focus will be on dry (i.e. non-lubricated) frictionless contact between the rolling elements and raceways. One of the main complexities in developing a non-linear bearing model is determining the mutual approach between a rolling element and a raceway, which generally requires a thorough geometrical analysis of the bearing. Once an expression for the mutual approach is obtained, its derivative with respect to time can be computed relatively easily (if not analytically, then using finite differencing) and this information can potentially be used to include the effect of EHL damping in the model by including a linear viscous damper. Finally, frictional forces are assumed to only slightly alter the load distribution across the rolling elements and will therefore not be considered in 
the present work (the interested reader is referred to Gupta (2012) for the inclusion of frictional forces and moments in the bearing model).

\subsubsection{Two-Dimensional Bearing Models}

$2 \mathrm{D}$ bearing models are typically used when investigating the global dynamical behavior of a planar multibody system (or one that can be idealized as such), or when the axial forces and misalignment torques (i.e. torques about the in-plane axes) in the bearing are negligible. Most two-dimensional bearing models in literature are based on (all of) the following assumptions:

- The rolling elements and inner and outer rings have motions in the plane of the bearing only;

- All deformations occur according to the Hertzian theory of elasticity, plastic deformations are neglected, and there is no bulk deformation of the bearing's components;

- The cage ensures a constant angular separation between rolling elements, hence there is no interaction between the rolling elements;

- There is no slipping of the rolling elements as they roll over the raceways;

- The bearings are assumed to operate under isothermal conditions such that thermal effects like the expansion of rolling elements and raceways and the change of lubricant viscosity can be neglected.

The assumptions of two-dimensional bearing modelling most naturally apply to bearings that are characterized by point contacts, such as ball bearings. Nevertheless, two-dimensional models have been used to model roller bearings as well, with only minor changes to the mathematical framework, as discussed below.

One of the distinguishing features of the various planar bearing models is the way the contact interactions and the inertial properties of the rolling elements are modelled. In a static bearing model (Fig. 9.5a), each rolling element (and the contact

(a)

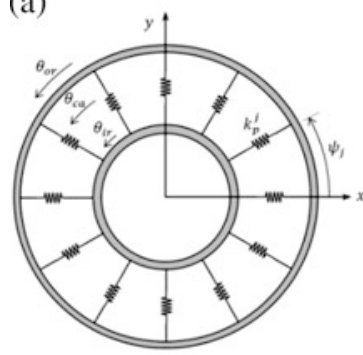

(b)

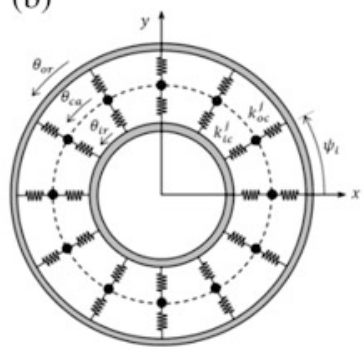

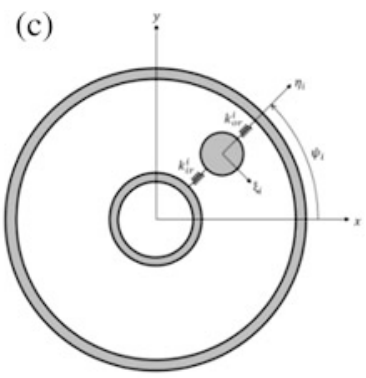

Fig. 9.5 2D bearing models: (a) static bearing model, (b) quasi-static bearing model, (c) dynamic bearing model 
interactions it has with both the inner and outer raceways) is represented by a single non-linear spring having zero stiffness when the spring is stretched. In this case, all dynamic effects that are due to the motion of the rolling elements (i.e. inertial and centrifugal forces) are neglected. Centrifugal effects are taken into account by the quasi-static bearing model (Fig. 9.5b) where each contact interaction is represented by a non-linear spring (i.e. two springs in series for each rolling element), and a centrifugal force proportional to the cage's angular velocity is applied at the interconnection between the two springs. This force acts to increase the clearance between the inner raceway and the rolling elements, and therefore alters the contact forces applied to the respective raceways (which are equal in magnitude in the case of the static bearing model). In addition to the series arrangement of non-linear springs, the dynamic bearing model (Fig. 9.5c) takes into account the mass of each rolling element and adds one degree of freedom per rolling element to the bearing's equations of motion. The latter are typically obtained by substituting the bearing's kinetic and potential energies into Lagrange's equation of motion, and need to be integrated in time simultaneously with the equations governing the dynamic behavior of the remainder of the multibody system.

Irrespective of the type of bearing model that is used, one critical aspect of modelling bearings, both in planar and spatial modelling, is the computation of the contact stiffness of the interaction between a rolling element and a raceway. The contact stiffness appears in the load-displacement relationship of the contact interaction, which has the form of the power law $Q=K_{c} \delta^{p}$, where $Q$ is the contact load, $K_{c}$ is the contact stiffness, $\delta$ is the mutual approach between rolling element and raceway, and $p$ is the exponent - the latter is equal to $3 / 2$ for point contacts according to Hertz's theory and is usually taken to be $10 / 9$ in the case of line contact (de Mul et al. 1989b). The contact stiffness $K_{c}$ depends on the contact geometry (radii of curvature of the contact surfaces) and the material properties (Young's modulus and Poisson's coefficient), and closed-form expressions are readily available in e.g. Harris and Kotzalas (2006). Because of the different geometries of the inner and outer raceways, the raceways typically have different contact stiffnesses.

Technical details on the mathematical derivation and implementation of twodimensional bearing models are readily available in literature. Static bearing models including the effects of non-linear contact stiffness, varying number of rolling elements in contact and radial clearance were used in Abbes et al. (2011), Kappaganthu and Nataraj (2011), and Xu and Li (2012) to study the dynamic behavior of respectively a pair of helical gears supported by ball bearings, a rotorbearing system and a slider-crank including a ball bearing joint. The inclusion of centrifugal effects in quasi-static bearing models is discussed in Hamrock and Anderson (1983), which includes a comprehensive overview of bearing modelling and all related aspects. Furthermore, an investigation of the influence of centrifugal effects is included in Liew et al. (2001). Dynamical two-dimensional ball bearing models were used in Harsha (2005) and Dougdag et al. (2012) to investigate the influence of bearing defects on system dynamics. Leblanc et al. (2009) developed 
a dynamical two-dimensional roller bearing including the influence of EHL and contact interactions with the cage.

\subsubsection{Three-Dimensional Bearing Models}

3D bearing models offer far greater insight into the internal bearing dynamics than their two-dimensional counterparts. They are typically used when the dynamic performance of the bearings are at the heart of the investigation, or when a threedimensional bearing representation is necessary to accurately grasp the global system behavior, e.g. when axial forces and/or misalignment torques are expected to play an important role. While compared to the overall computational cost of general multibody systems the three-dimensional bearing models are not significantly less efficient than the 2D models, the numerical implementation of these models is often considerably more involved. Due to the fundamental differences in the nature of the contact interactions (point vs. line contact), different mathematical models are typically used to model ball and roller bearings.

\subsubsection{Ball Bearings}

In the case of point contacts, all internal bearing forces and moments originate from simple Hertzian point contact interactions (between rolling elements and raceways) that are described by the same load-displacement relationship given in Sect 9.3.2. The complexity of three-dimensional ball bearing models is therefore solely related to the rather complex geometry and kinematics of three-dimensional ball bearings.

The first to investigate three-dimensional ball bearing models in a systematic way was Jones (1960). He formulated a quasi-static three-dimensional ball bearing model that included the influence of centrifugal forces and gyroscopic moments. The model consists of five nonlinear, simultaneous equilibrium equations (corresponding to the five coordinates that are constrained by the bearing, cf. Sect. 9.3.1) that need to be linearized and solved iteratively by the Newton-Raphson method. The complexity of the model lies mainly in determining the internal bearing forces and moments, which are expressed w.r.t. a reference frame that is aligned with the shaft (or bearing inner ring), leading to rather cumbersome expressions, especially when the influence of quadratic velocity terms is included.

In response to the complexity of the Jones model, Gupta (2012) developed a dynamical ball bearing model (in which each ball bearing was assigned with a number of degrees of freedom), arguing that despite the increased computing effort in obtaining a solution (through integration of the equations of motion), the dynamic model provides a significant reduction in the overall design costs of the model (as the influence of centrifugal and gyroscopic effects follows naturally from the bearing's equation of motion). Furthermore, the dynamic model is free of the typical convergence problems that are associated with solving equilibrium solutions 
in quasi-static bearing models, and therefore are better suited for including lubricant behavior (Gupta 2012).

Another simplification of the model of Jones was provided by de Mul et al. (1989a), who developed a quasi-static bearing model in five degrees of freedom and including centrifugal forces. The formulation of the model is less cumbersome than the model of Jones and lends itself better for computer implementation. Assuming no circumferential motion of the rolling element at equilibrium, the equilibrium equations for each roller element are formulated in a plane (two translations and one rotation) at the rolling element level, and are then transformed to the central bearing reference frame through a 3-by-5 transformation matrix. The mutual approach between rolling element and raceway is obtained through a comparatively simple two-dimensional geometric analysis, and closed-form expressions for the bearing's global stiffness matrix are provided.

\subsubsection{Roller Bearings}

The classical Hertzian theory is usually not valid for roller contact. This can be either because of the dimension of the contact area that exceeds the assumption of Hertz, due to noncircular contact profiles, or due to high loads making the contact ellipse being truncated at the end of the rollers. These types of problems are referred to as non-Hertzian contact problems and there is, unfortunately, no exact and explicit relationship available for expressing the load as a function of the deflection. Many authors, such as Lundberg and Sjövall (1958), Palmgren (1959), and Young and Budynas (2002), have addressed these types of problems based on half-space theory and experiments to find an, often implicit, relationship between the load and the deformation. These relationships are often found in idealized contact situations (e.g. no misalignments, close to half-space conditions) and sometimes even profiled roller geometries.

More recently, numerical techniques are adopted to account e.g. for misaligned rollers. Some authors numerically integrate the Boussinesq's half-space theory to iteratively obtain the required solution (Reusner 1977; Andréason 1973). Others apply more simple techniques (Harris and Kotzalas 2006; Andréason 1973), however less accurate, for computational reasons in case that multiple contacts need to be solved simultaneously. In the so-called slicing technique, the contact length is sliced into a number of laminae. Each slice is given a fraction of the total roller stiffness, which is often based on some of the above mentioned load-deflection relations. A distinction can be made in whether each slice is treated separately, and thus having no effect on the deflection of adjacent slices, or treated in a combined way, and thus having a mutual influence. In the former case, pressure concentrations cannot be taken into account. Nevertheless, the problem is less expensive to solve. In the other case, where the deflection of one slices depends on the deflection of other slices, iterative procedures are required. Teutsch and Sauer (2004) propose a method in which the optimum between accuracy and efficiency is searched for. 
The modelling of 3D roller bearings is conceptually similar to ball bearings; the mutual approach between the contacting bodies has to be found such that the loaddeflection relation can be applied and the contact forces computed. As mentioned above, the slicing technique is often used to discretize the roller into a set of laminae. Consequently, the mutual approach of each slice to the bearing raceway should be determined by a (kinematic) model.

As for ball bearings, many authors have provided various levels of complexity. Gupta (2012) followed his own approach for ball bearings and assigned again degrees-of-freedom to the rollers. The slicing technique is adopted to compute the roller contact forces. In models such as those of de Mul et al. (1989b) and Andréason (1973), among many others, the equilibrium equations are again determined in a two-dimensional plane at rolling element level and include the centrifugal and gyroscopic forces in a quasi-static way. de Mul et al. (1989b) refer next to the slicing technique also to a more sophisticated technique to model non-Hertzian contact in a general way that was presented in de Mul et al. (1986).

To end this section, it should be noted that rollers are profiled by the bearing manufacturers, in order to optimize the stress distribution over the rollers for a particular loading condition and to avoid high edge stresses that can substantially exceed the computed stresses in case of separately treated slices. The knowledge of the profile, as for the geometry of the raceways and rolling elements in bearings, is key for an accurate prediction of the contact stresses and the stiffness of a bearing.

\subsubsection{Bearings with Distributed Flexibility}

The above listed solutions pertain to bearings that have rigidly supported rings. The considered deflections are the local Hertzian deformations and are based on the assumptions presented in 9.2.2. In some applications, however, the influence of the supporting structure's and ring's flexibility can be decisive for the bearing's behavior as it might affect, for instance, the load distribution over the rolling elements. Consequently, the global (or bulk) deformations such as the bending of the bearing housing cannot always be neglected.

An obvious way to tackle this modelling problem is to use the Finite Element (FE) method to include and combine both the local nonlinear contact deflections and the global flexibility of the ring and supporting structure. In addition, the influence of e.g. materials, manufacturing errors, lubrication, and cage effects has been investigated by adding those effects to (nonlinear) FE codes. A brief overview of such codes is given in Qian (2013). Although these (nonlinear) FE approaches have proven their value for many high technology applications, they suffer from some severe disadvantages for drivetrain modelling. First, since the contact interaction (or mutual approach) between the rolling elements and the raceways can generally not be sought purely analytically as for the techniques listed in the previous sections, some standard contact detection algorithms are obliged to search for contact in all possible contact elements. This process is often the most time-consuming process 
in dynamic contact simulations. It should however be mentioned that, generally, the known (undeformed) geometry can in fact be exploited to reduce the number of possible contact elements for a specific rolling element. A second disadvantage is that one has to rely on FE models with a highly refined mesh at the contact zone and thus a large number of nodes in order to capture the nonlinear behavior of the Hertzian contact with a reasonable accuracy. The resulting models have therefore an extremely high number of degrees-of-freedom (DOFs). For time-domain analyses of gearboxes and drivetrains, where it is not uncommon to have multiple bearings and gears, these models result in unacceptable simulation times.

Model order reduction schemes can be introduced to lower the dimensionality of the problem to alleviate above mentioned computational burden with a minimal loss of accuracy. However, traditional model order reduction techniques typically become inefficient for models with time-varying load locations, as is the case for the moving rolling elements in a bearing. In fact, to obtain a favorable accuracy on displacement and stress levels one has to include so-called static modes for each of the interacting nodes in the reduction space, as it is likely that almost every node positioned on the raceway of the bearing can be loaded by the rolling elements. This action might result in a poor reduction in terms of DOFs.

To deal with the latter problem, some advanced model order reduction techniques have been developed to accurately simulate problems with time-varying load locations, such as gears and bearings. A statically complete procedure is provided by the Static Modes Switching (SMS) method (Heirman et al. 2011), which has been applied to the gear contact problem in Tamarozzi et al. (2013). SMS makes use of a discontinuously changing reduction basis, of minimal dimension, by taking advantage of the fact that each time step only a few out of many interface DOFs are loaded simultaneously. The technique has been modified in Tamarozzi et al. (2014) to obtain a continuously time-varying reduction space by use of a parametric relationship describing the location of externally applied moving loads, further referred to as Static Mode Sliding $\left(\mathrm{SMS}^{2}\right)$. It has been shown that the price to pay for the very compact reduction basis is the evaluation of some extra terms in the equations of motion. This model order reduction strategy is later extended and applied for the simulation and prediction of the behavior of an indirect dynamic bearing force measurement setup (Fiszer et al. 2015b) and for efficient timesimulation of gear pairs (Blockmans et al. 2015). In Blockmans et al. (2015), the performance of the novel technique is demonstrated by simulating a dynamic gear contact problem and comparing results against traditional model reduction techniques as well as commercial nonlinear finite element software.

Although the computational burden is alleviated by use of the model order reduction techniques, a highly refined mesh at the contact zone is still required to capture the nonlinear behavior of the Hertzian contact. The latter retains the need for a large number of so-called static modes to be computed in a pre-processing step, despite the fact that only few are active at a certain moment in time during the simulation. 
Some authors have proposed to combine analytic solutions with the FE method, (Wensing 1998; Andersson and Vedmar 2003; Vijayakar 1991; Fiszer et al. 2015a), thereby eliminating the need for highly refined meshes at the contact zone. The main advantage of such a semi-analytic strategy lies in the accurate contact description that combines the nonlinear effects present at the contacting interface with a standard FE formulation in those regions in which nonlinear effects are not prominent. Therefore, coarser FE meshes can be used; these meshes should only accurately represent the more global bulk deformation.

In the semi-analytic strategy presented by Wensing (1998), the total deformation is separated in the bulk deformation, represented by a reduced order model, and the nonlinear local Hertzian deflections at the contact zone. The multitude of static shape vectors is replaced by a comparatively small set of global constraint modes to capture the effect of the bulk flexibility of the rings, supporting structure and the mounting conditions on the behavior of the bearings. The global constraint modes are determined by use of Chebyshev polynomials, which are also utilized as contact geometry. These analytic surfaces are then used to determine the mutual approach between the ring and the rolling element to compute the contact forces based on Hertzian contact theory.

The approach presented in Fiszer et al. (2015a) offers an equivalent semi-analytic strategy. Nevertheless, the global constraint modes, that are used to reduce the FE model representing the bulk deformation, are replaced by global attachment modes. The latter allows for a one-to-one relation between the contact load and the static mode, such that a parametric relationship describing the contact location can be defined and the efficient model reduction technique $\mathrm{SMS}^{2}$ becomes applicable. In addition, the Chebyshev polynomials that represent smooth interacting raceways are replaced by NURBS or B-spline surfaces. It is shown in Fiszer et al. (2015b) that by selecting an appropriate basis for the B-splines, the interacting surface can be composed by a linear combination of the control points, using the modal participation factors (or reduced elastic coordinates) of the affected mode shapes during simulations. These offline computed B-splines or NURBS are therefore referred to as NURBS-modes.

\subsection{Conclusions: Towards Unified Wind Turbine Gearbox Models}

Sections 9.2 and 9.3 underlined some key aspects regarding the behavior and the modelling of wind turbine drivetrains with a specific focus on bearings. It goes without saying that another major component that needs to be accurately simulated within drivetrains, are gears. Helical stages and planetary helical stages are mostly of relevance in this framework. Similarly to bearings, gears are also complex components in which accurate geometry, material properties and lubrication play an important role. They can also be simulated with different levels of complexity 
ranging from analytical models and lumped parameter models (Özgüven and Houser 1988; Cai and Hayashi 1994; Kuang and Yang 1992; Velex 2012; Kahraman 1993; Eritenel and Parker 2012; Kahraman 1994; Lin and Parker 1999; Andersson and Vedmar 2003) up to fully flexible non-linear contact simulations (Tamarozzi et al. 2013; Blockmans et al. 2015; Ziegler et al. 2006; Parker et al. 2000). The literature about gear modelling and analysis is as rich as the one for bearings and it is out of the scope of this chapter to provide a detailed review.

While the modelling of single components can be interesting for troubleshooting of localized problems in a wind turbine gearbox, these are seldom enough to draw conclusions regarding the behavior of the full drivetrain. In order to benefit from the different levels of complexity in which gears and bearings can be represented, there is a strong need for coupling them to system-level simulation tools such as e.g. multibody and flexible multibody models. Multibody simulation is an established technology that is aimed to investigate the dynamic behavior of systems of interconnected bodies. Within this type of models it is possible to connect different body representations (e.g. rigid or flexible) by making use of idealized joints or force elements that can represent localized stiffness and damping with different levels of complexity. Examples of wind turbine drivetrains modelled through multibody simulation can be found in Helsen (2012) and Vanhollebeke (2015).

Multibody models with idealized joints or constraints form a set of highly nonlinear Differential Algebraic Equations (DAE). For relatively simple systems with mainly rigid bodies, computational strategies exist to perform dynamic transient simulations in a relatively short time-frame. Depending on the phenomena that need to be investigated, bearings and gears models can be included in multibody models with different levels of complexity. In order to achieve drivetrain models that are of wide applicability and address different design questions some research effort still needs to be focused on several open topics as e.g.:

- Scalability of different model complexities for bearings and gears to be included in system level simulation. In particular bearings and gears can be idealized in different ways but all the different model types should be able to interact with their surrounding environment. Depending on the model complexity adopted for the different components, these interactions can happen at localized or distributed interfaces. As an example, a gear or a bearing can be modelled in three dimensions as a $6 \times 6$ stiffness matrix that interacts with shafts and housing through forces and torques acting at localized interface points. On the other extreme, flexible gear models and bearings interact with their surrounding structure through distributed FE interface nodes. The inherently different interface behavior of the modelling strategies makes it difficult to create a single drivetrain model that is scalable for different levels of complexity depending on the target use. The inclusion of 3D modelling capabilities within natively 1D environments such as Matlab Simulink, SISW Imagine. Lab Amesim, Dymola, etc.... could potentially alleviate these difficulties and is topic of current research. 
- Advanced drivetrain models including e.g. flexible housing deformation, contact mechanics models for gears and bearings, spline connection models, etc.... can easily become computationally cumbersome. Modern linear and non-linear model order reduction techniques are one of the main research fields from which wind turbine drivetrain modelling could benefit.

Sections 9.1-9.3 summarized a few key focal points regarding drivetrain modelling in a wind turbine context with specific focus on state-of-the-art of bearing modelling. The discussion should not be considered as exhaustive but underlines a few important items to be considered when analyzing and simulating drivetrains. The improvements of components modelling methods (e.g. bearing and gears) and scalable inclusion in system-level simulation tools for accurate and affordable drivetrain analysis remain (partially) open challenges where further research efforts needs to be focused.

Acknowledgements The authors would like to gratefully acknowledge the European Commission for their support of the MAREWINT research project (GA 309395). Additionally, the IWT Flanders within the OPTIWIND project and the Research Fund KU Leuven for the IDO_WIND project are kindly acknowledged for their support.

Open Access This chapter is distributed under the terms of the Creative Commons AttributionNonCommercial 4.0 International License (http://creativecommons.org/licenses/by-nc/4.0/), which permits any noncommercial use, duplication, adaptation, distribution and reproduction in any medium or format, as long as you give appropriate credit to the original author(s) and the source, provide a link to the Creative Commons license and indicate if changes were made.

The images or other third party material in this chapter are included in the work's Creative Commons license, unless indicated otherwise in the credit line; if such material is not included in the work's Creative Commons license and the respective action is not permitted by statutory regulation, users will need to obtain permission from the license holder to duplicate, adapt or reproduce the material.

\section{References}

Abbes MS, Hentati T, Maatar M et al (2011) Dynamic analysis of helical gears supported by rolling elements bearings. J Theor Appl Mech 41(1):33-50

Andréason S (1973) Load distribution in a taper roller bearing arrangement considering misalignment. Tribology 6(3):84-92. doi:10.1016/0041-2678(73)90241-8

Andersson A, Vedmar L (2003) A dynamic model to determine vibrations in involute helical gears. J Sound Vib 260(2):195-212

Blockmans B, Tamarozzi T, Naets F et al (2015) A nonlinear parametric model reduction method for efficient gear contact simulations. Int J Numer Methods Eng 102(5):1162-1191

Brandlein J, Eschmann P, Hasbargen L et al (1999) Ball and roller bearings: theory, design and application. Wiley, Chichester

Cai Y, Hayashi T (1994) The linear approximated equation of vibration of a pair of spur gears (theory and experiment). J Mech Des 116(2):558-564

Chen W, Alewine K (2010) Wind turbine generator failure modes analysis and occurrence. Paper presented at the Wind Power, Dallas, 24-26 May 2010 
de Mul JM, Kalker JJ, Fredriksson B (1986) The contact between arbitrarily curved bodies of finite dimensions. J Tribol 108(1):140-148

de Mul JM, Vree JM, Maas DA (1989a) Equilibrium and associated load distribution in ball and roller bearings loaded in five degrees of freedom while neglecting friction - part I: general theory and application to ball bearings. J Tribol 111(1):142-148

de Mul JM, Vree JM, Maas DA (1989b) Equilibrium and associated load distribution in ball and roller bearings loaded in five degrees of freedom while neglecting friction - part II: application to roller bearings \& experimental verification. J Tribol 111(1):149-155

Dietl P, Wensing J, van Nijen G (2000) Rolling bearing damping for dynamic analysis of multibody systems: experimental and theoretical results. Proc Inst Mech Eng K J Multi-Body Dyn 214:3343

Dougdag M, Ouali M, Boucherit $\mathrm{H}$ et al (2012) An experimental testing of a simplified model of a ball bearing: stiffness calculation and defect simulation. Meccanica 47(2):335-354

Eritenel T, Parker RG (2012) Three-dimensional nonlinear vibration of gear pairs. J Sound Vib 331(15):3628-3648

EWEA (2011) Upwind: design limits and solutions for very large wind turbines. In: European Wind Energy Association Wind Energy Association Reports. Available via EWEA. http:// www.ewea.org/fileadmin/files/library/publications/reports/UpWind_Report.pdf. Accessed 08 Apr 2016

Fiszer J, Tamarozzi T, Desmet W (2015a) A semi-analytic strategy for the system-level modelling of flexibly supported ball bearings. Meccanica. doi:10.1007/s11012-015-0318-6

Fiszer J, Tamarozzi T, Blockmans B et al (2015b) A time-dependent parametric model order reduction technique for modelling indirect bearing force measurements. Mech Mach Theory 83:152-174

Gegner J (2011) Tribological Aspects of Rolling Bearing Failures. In: Kuo CH (ed) Tribology lubricants and lubrication. InTech, Rijeka, pp 33-94

Grubin A (1949) Fundamentals of the hydrodynamic theory of lubrification of heavily loaded cylindrical surfaces. In: Ketova $\mathrm{KhF}$ (ed) Symposium on Investigation of the Contact of Machine Components, Moscow, 1949, Central Scientific Research Institute for Technology and Mechanical Engineering, vol 30. TsNIITMASh, Moscow, p 115

Guo Y, Keller J, LaCava W (2014) Planetary gear load sharing of wind turbine drivetrains subjected to non-torque loads. Wind Energy 18(4):757-768

Gupta PK (2012) Advanced dynamics of rolling elements. Springer, New York

Hamrock BJ, Anderson WJ (1983) Rolling-element bearings. NASA Reference Publication 1105. National Aeronautics and Space Administration Publications. Available via NASA NTRS. http://ntrs.nasa.gov/archive/nasa/casi.ntrs.nasa.gov/19830018943.pdf. Accessed 08 Apr 2016

Harris TA, Kotzalas M (2006) Advanced concepts of bearing technology: rolling bearings analysis. Taylor and Francis, New York

Harsha SP (2005) Non-linear dynamic response of a balanced rotor supported on rolling element bearings. Mech Syst Signal Process 19(3):551-578

Heirman GHK, Tamarozzi T, Desmet W (2011) Static modes switching for more efficient flexible multibody simulation. Int J Numer Methods Eng 87(11):1025-1045

Helsen J (2012) The dynamics of high power density gear units with focus on the wind turbine application. Dissertation, KU Leuven

ISO (2007) ISO 281:2007 Rolling bearings - dynamic load ratings and rating life. ISO, Geneva

Jacobs W (2014) Experimental analysis of the dynamic characteristics and lubricant film of a ball bearing under combined static and dynamic load. Dissertation, KU Leuven

Jones AB (1946) New departure engineering data; analysis of stresses and deflections. Bristol Conn, New Departure, Division General Motors Corp, Chicago

Jones AB (1960) A general theory for elastically constrained ball and radial roller bearings under arbitrary load and speed conditions. J Basic Eng 82(2):309-320

Kahraman A (1993) Effect of axial vibrations on the dynamics of a helical gear pair. J Vib Acoust 115(1):33-39

Kahraman A (1994) Planetary gear train dynamics. J Mech Design 116(3):713-720 
Kappaganthu K, Nataraj C (2011) Nonlinear modeling and analysis of a rolling element bearing with a clearance. Commun Nonlinear Sci 16(10):4134-4145

Kuang JH, Yang YT (1992) An estimate of mesh stiffness and load sharing ratio of a spur gear pair. In: The 1992 ASME design technical conferences - 6th international power transmission and gearing conference, Scottsdale, September 1992. Advancing Power Transmission into the 21st Century, vol 1. ASME, New York, p 1

Leblanc A, Nelias D, Defaye C (2009) Nonlinear dynamic analysis of cylindrical roller bearing with flexible rings. J Sound Vib 325(1):145-160

Li Y, Billington S, Kurfess C et al (1999) Adaptive prognostics for rolling element bearing condition. Mech Syst Signal Process 13(1):103-113

Liew A, Feng NS, Hahn EJ (2001) Transient rotordynamic modeling of rolling element bearing systems. In: Abstracts of the ASME Turbo Expo 2001: Power for Land, Sea, and Air, American Society of Mechanical Engineers, New Orleans, 4-7 June 2001

Lin J, Parker RG (1999) Analytical characterization of the unique properties of planetary gear free vibration. J Vib Acoust 121(3):316-321

Lundberg G, Sjövall H (1958) Stress and deformation in elastic contacts. Institution of Theory of Elasticity and Strength of Materials, Gothenburg

Mitsuya Y, Sawai H, Shimizu M, Aono Y (1998) Damping in vibration transfer through deepgroove ball bearing. J Tribol 120:413-420

Muro H, Tsushima N (1970) Microstructural, microhardness and residual stress changes due to rolling contact. Wear 15:309-330

Nierlich W, Gegner J (2011) Material response bearing testing under vibration loading. In: Beswick J (ed) Selected Technical Papers STP 1548 Bearing Steel Technologies. Ninth International Symposium on Bearing Steel Technologies, November 2011. Advances in Rolling Contact Fatigue Strength Testing and Related Substitute Technologies, vol 9. ASTM, New York

Nonato F, Cavalca K (2012) Local linear approximation for the stiffness characteristics of elastohydrodynamic point contacts. In: Abstracts of the 10th international conference on vibrations in rotating machinery, IMechE, London, 11-13 Sep 2012

Özgüven HN, Houser DR (1988) Mathematical models used in gear dynamics-a review. J Sound Vib 121(3):383-411

Palmgren A (1959) Ball and roller bearing engineering. SH Burbank, Philadelphia

Park YJ, Lee GH, Song JS, Nam YY (2013) Characteristic analysis of wind turbine gearbox considering non-torque loading. J Mech Des 135(4):044501-044508. doi:10.1115/1.4023590

Parker RG, Agashe V, Vijayakar SM (2000) Dynamic response of a planetary gear system using a finite element/contact mechanics model. J Mech Des 122(3):304-310

Peeters J (2006) Simulation of dynamic drive train loads in a wind turbine. Dissertation, KU Leuven

Qian W (2013) Dynamic simulation of cylindrical roller bearings. Dissertation, RWTH Aachen University

Reusner H (1977) Druckflächenbelastung und Oberflächenverschiebung im Wälzkontakt von Rotationskörpern. Dissertation TH Karlsruhe

Tamarozzi T, Ziegler P, Eberhard P et al (2013) Static modes switching in gear contact simulation. Mech Mach Theory 62:69-106. doi:10.1016/j.mechmachtheory.2013.01.006

Tamarozzi T, Heirman GH, Desmet W (2014) An on-line time dependent parametric model order reduction scheme with focus on dynamic stress recovery. Comput Methods Appl Mech Eng 268:336-358

Teutsch R, Sauer B (2004) An alternative slicing technique to consider pressure concentrations in non-Hertzian line contacts. J Tribol 126(3):436-442

Vanhollebeke F (2015) Dynamic analysis of a wind turbine gearbox: towards prediction of mechanical tonalities. Dissertation, KU Leuven

Velex P (2012) On the modelling of spur and helical gear dynamic behaviour. Cornell University Library: Classical Physics. Available via arXiv. http://arxiv.org/pdf/1204.2636v1 preprint arXiv:1204.2636. Accessed 07 Apr 2016 
Vijayakar S (1991) A combined surface integral and finite element solution for a three-dimensional contact problem. Int J Numer Methods Eng 31(3):525-545

Wensing J (1998) On the dynamics of ball bearings. Dissertation, University of Twente

Wijnant Y (1998) Contact dynamics in the field of elastohydrodynamic lubrication. Dissertation, University of Twente

Xu LX, Li YG (2012) An approach for calculating the dynamic load of deep groove ball bearing joints in planar multibody systems. Nonlinear Dyn 70(3):2145-2161

Young WC, Budynas RG (2002) Roark's formulas for stress and strain, vol 7. McGraw-Hill, New York

Ziegler P, Eberhard P, Schweizer B (2006) Simulation of impacts in geartrains using different approaches. Arch Appl Mech 76:537-548

Zwirlein O, Schlicht H (1980) Werkstoffanstrengung bei Wälzbeanspruchung - Einfluss von Reibung und Eigenspannungen. Werkstofftechnik 1:11-14 